M. Alonso ${ }^{1}$

E. Camus ${ }^{2}$

J. Rodriguez Diego ${ }^{1}$

L. Bcrtaudic̀re ${ }^{3}$

J.C. Tatareau ${ }^{4}$

J.M. Liabeuf ${ }^{5}$

\title{
Situation actuelle des hémoparasitoses bovines en Martinique (Antilles françaises)
}

\begin{abstract}
ALONSO (M.), CAMUS (E.), RODRIGUEZ DIEGO (J.), BERTAU. DIERE (L.), TATAREAU (J.C.), LIABEUF (J.M.). Situation actuelle des hémoparasitoses bovines en Martinique (Antilles françaises). Revue Élev. Méd. vét. Pays trop., 1992, 45 (1) : 9-14

Une enquête sérologique par immunofluorescence indirecte pour les hahésioses bovines (Babesia bovis et $\boldsymbol{B}$. bigemina) et par agglutination pour l'anaplasmose bovine, indique que ces hémoparasitoses sont largement répandues en Martinique. Les séroprévalences élevées $(62$ p. 100 pour $B$. bovis, 52 p. 100 pour $B$. bigemina et 43 p. 100 pour Anaplasma marginale) font penser à une situation épizootiologique instable pour ces trois hémoparasitoses mais le faible nombre de frottis observés ne permet pas d'apprécier leur incidence clinique. Les deux Card tests américain et cubain donnent des résultats pratiquement identiques dans la détection d'anticorps anti-A. marginale. Theileria mutans est signalé pour la première fois en Martinique. Quant à la trypanosomose à Trypanosoma vivax, elle a disparu de l'île cliniquement et sérologiquement. Mots clés : Babesia bovis - Babesia bigemina - Anaplasma marginale - Theileria mutans - Trypanosoma vivax - Sérologie - Immunodiagnostic - Card test - Martinique.
\end{abstract}

\section{INTRODUCTION}

La première étude exhaustive sur les hémoparasitoses bovines en Martinique est due à MOREL (12) ; il signale que "la piroplasmose bovine à Babesia bigemina existe en Guadeloupe et Martinique", ainsi que "la disparition probable de Trypanosoma vivax observé entre 1928 et 1938 (5)". II récolte des Boophilus microplus dans toute l'île. En 1984, une enquête sérologique était réalisée par CAMUS (non publiée) sur la cowdriose (soupçonnée avec 10 p. 100 de bovins séropositifs), l'anaplasmose (39 p. 100 de bovins séropositifs) et les babésioses (22 p. 100 de bovins séropositifs vis-à-vis de $B$. bovis et 16 p. 100 vis-à-vis de $B$. bigemina).

Or, la pathologie bovine est dominée en Martinique par la dermatophilose (3) qui fait oublier les hémoparasitoses.

1. Centro Nacional de Sanidad Agropecuaria (CENSA), Apto 10 , San José de las Lajas, La Habana, Cuba.

2. CIRAD-EMVT, BP 1232, 97184 Pointe-à-Pitre Cedex, Guadeloupe.

3. Direction des Services vétérinaires (DSV), BP 671, 97262 Fortde-France Cedex, Martinique

4. Établissement départemental de l'Élevage (EDE), BP 312, 97286 Lamentin Cedex 02, Martinique.

5. Centre commercial Place d'Armes, 97232 Lamentin, Martinique.

Reçu le 22.10.91, accepté le 18.2.1992.
On a, dans ce contexte, réalisé une étude préliminaire avec pour objectifs :

- étudier la séroprévalence de l'anaplasmose et des babésioses en 1989, ainsi que leur incidence clinique ;

- comparer deux card tests pour le diagnostic sérologique de l'anaplasmose, l'un américain, l'autre cubain ;

- vérifier la réalité de la disparition de Trypanosoma vivax;

- rechercher la présence d'autres parasites tels que Theileria mutans.

\section{MATÉRIEL ET MÉTHODES}

\section{Sérums}

Ils ont été récoltés par des techniciens de la Direction des Services vétérinaires (DSV) et de l'Établissement départemental de l'Élevage (EDE) à l'occasion d'une enquête sur la situation d'Amblyomma variegatum : 227 troupeaux de bovins ont été examinés dans tout le département et, dans chaque troupeau, un animal était choisi au hasard pour un prélèvement de sang. Une fiche d'enquête indiquait :

- les tiques et les modalités de la lutte: présence d'A. variegatum, rythme et produit de détiquage ;

- les caractéristiques de l'animal : race, âge, sexe, présence de tiques.

\section{Méthodes de diagnostic sérologique}

\section{Anaplasmose}

Une méthode américaine d'agglutination sur plaque (Anaplasmosis Card test ${ }^{\mathrm{N} U *}$ ) a été comparée à une autre, mise au point à Cuba (Anacen ${ }^{\mathrm{ND}}$, Davinlab s/c CENSA ${ }^{\star \star}$ ).

Les deux antigènes sont préparés à partir des globules rouges parasités ; dans le premier cas ils sont pressés avec une "presse française" (2) et dans le deuxième cas ils sont soumis à des ultra-sons (1).

\footnotetext{
* Hynson, Westcott \& Dunning, Baltimore, Maryland 21201, États-Unis.
}

* Centro Nacional de Sanidad Agropecuaria. 


\section{Alonso E. Camus J. Rodriguez Diego L. Bertaudière J.C. Tatareau J.M. Liabeuf}

\section{Babésioses - Trypanosomose}

Les anticorps contre ces maladies ont été recherchés par un test classique d'immunofluorescence indirecte.

Les souches fournies par le Pr G. UILENBERG (CIRADEMVT) provenaient pour B. bovis d'lsraël et pour B. bigemina du Nigeria. Elles ont été inoculées à deux veaux Frison splénectomisés. A l'acmé parasitaire, le sang a été prélevé et directement utilisé pour faire des frottis, séchés, fixés dans de l'acétone et conservés sous plastique à $-20^{\circ} \mathrm{C}$.

La souche de $T$. vivax a été isolée en Guyane par R. LANCELOT et S. FERENC (10) sur un bovin, passée sur une chèvre immunodéprimée pour éliminer les Babesia et augmenter la parasitémie. La technique de préparation des antigènes est identique à celle des Babesia. La dilution seuil est de 1:80 pour Babesia et 1:160 pour $T$. vivax.

\section{Frottis}

Des frottis de sang et de cerveau prélevés sur des bovins malades ou morts et suspects d'être atteints de cowdrio- se, de babésiose ou d'anaplasmose ont été colorés par la méthode de coloration rapide (RAL $555^{\mathrm{ND}}$ ).

\section{Analyse statistique des résultats}

Elle a été réalisée par analyse factorielle de correspondance et $\chi^{2}$.

\section{RÉSULTATS}

\section{Enquête sérologique}

Les résultats figurent dans les tableaux I et II avec les différents paramètres explicatifs : localisation géographique, race, âge, mode de détiquage.

Aucun sérum n'a présenté d'anticorps vis-à-vis de Trypanosoma vivax sur les 227 troupeaux d'enquêtes concernant 27 communes soit 2 à 3 p. 100 de l'ensemble du cheptel bovin. Deux cent soixante-

TABLEAU I Pourcentage de bovins séropositifs vis-à-yis d'Anaplasma marginale, Babesia bovis et B. bigemina. Variation du pourcentage en fonction de divers facteurs.

\begin{tabular}{|c|c|c|c|c|}
\hline & & $\begin{array}{c}\text { Anaplasma } \\
\text { marginale }\end{array}$ & $\begin{array}{c}\text { Babesia } \\
\text { bovis }\end{array}$ & $\begin{array}{c}\text { Babesia } \\
\text { bigemina }\end{array}$ \\
\hline \multirow{3}{*}{ Localisation } & Sud & $62 / 156=40$ p. cent & $86 / 156=55$ p. cent & $76 / 156=49$ p. cent \\
\hline & Nord & $46 / 94=49$ p. cent & $68 / 94=72$ p. cent & $55 / 94=59$ p. cent \\
\hline & $x^{2}$ & NS & $<1 \%$ TS & NS \\
\hline \multirow{4}{*}{ Race } & Européenne & $10 / 23=43$ p. cent & $12 / 23=52$ p. cent & $11 / 23=48$ p. cent \\
\hline & Brahman & $20 / 51=39$ p. cent & $31 / 51=61 \mathrm{p}$. cent & $28 / 51=55$ p. cent \\
\hline & Créole-Brahman & $62 / 135=46$ p. cent & $91 / 135=67$ p. cent & $74 / 135=55$ p.cent \\
\hline & Créole & $15 / 37=41$ p. cent & $10 / 37=27$ p. cent & $9 / 37=24$ p. cent \\
\hline \multirow{5}{*}{ Age (ans) } & $<1$ & $9 / 21=42$ p. cent & $13 / 21=61$ p. cent & $10 / 21=48$ p. cent \\
\hline & $1-2$ & $17 / 51=33$ p. cent & $32 / 51=62$ p. cent & $27 / 51=53$ p. cent \\
\hline & $3-4$ & $25 / 65=38$ p. cent & $47 / 65=72$ p. cent & $39 / 65=60$ p. cent \\
\hline & $5-6$ & $26 / 47=63$ p. cent & $28 / 47=59$ p. cent & $24 / 47=51$ p. cent \\
\hline & $>6$ & $23 / 41=56$ p. cent & $20 / 41=49$ p. cent & $20 / 41=49$ p. cent \\
\hline \multirow{2}{*}{ Détiquage } & DSV & $57 / 141=40$ p. cent & $80 / 141=57$ p. cent & $71 / 141=50$ p. cent \\
\hline & Autres Sud & $5 / 15=33 p$. cent & $6 / 15=40$ p. cent & $5 / 15=33 p$. cent \\
\hline Total & & $108 / 250=43$ p. cent & $154 / 250=62$ p. cent & $131 / 250=52$ p. cent \\
\hline
\end{tabular}


TABLEAU II Distribution par communes de bovins séropositifs vis-à-vis d'Anaplasma marginale, Babesia bovis et $\mathrm{B}$. bigemina.

\begin{tabular}{|c|c|c|c|}
\hline & $\begin{array}{c}\text { Anaplasma } \\
\text { marginale }\end{array}$ & $\begin{array}{c}\text { Babesia } \\
\text { bovis }\end{array}$ & $\begin{array}{c}\text { Babesia } \\
\text { bigemina }\end{array}$ \\
\hline Sud & & & \\
\hline Lamentin & $5 / 16=0,31$ & $10 / 16=0,62$ & $11 / 16=0,69$ \\
\hline Ducos & $8 / 11=0,73$ & $10 / 11=0,91$ & $5 / 11=0,45$ \\
\hline Rivière-Pilote & $4 / 8=0,50$ & $1 / 8=0,12$ & $1 / 8=0,12$ \\
\hline Trois-llets & $5 / 5=1,00$ & $3 / 5=0,60$ & $2 / 5=0,40$ \\
\hline Diamant & $6 / 7=0,86$ & $5 / 7=0,71$ & $5 / 7=0,71$ \\
\hline Sainte-Luce & $3 / 11=0,27$ & $2 / 11=0,18$ & $1 / 11=0,09$ \\
\hline Le Marin & $3 / 11=0,23$ & $10 / 13=0,77$ & $9 / 13=0,69$ \\
\hline Sainte-Anne & $8 / 28=0,29$ & $17 / 28=0,61$ & $10 / 28=0,36$ \\
\hline Vauclin & $9 / 29=0,31$ & $16 / 29=0,55$ & $17 / 29=0,59$ \\
\hline Rivière-Salée & $6 / 12=0,50$ & $3 / 12=0,25$ & $7 / 12=0,58$ \\
\hline François & $6 / 13=0,46$ & $7 / 13=0,54$ & $6 / 13=0,46$ \\
\hline Nord & & & \\
\hline $\begin{array}{l}\text { Robert- } \\
\text { St-Joseph- } \\
\text { St-Denis }\end{array}$ & $3 / 11=0,27$ & $8 / 11=0,73$ & $7 / 11=0,64$ \\
\hline $\begin{array}{l}\text { Case-Pilote- } \\
\text { Belle-Fontaine }\end{array}$ & $6 / 9=0,67$ & $8 / 9=0,89$ & $7 / 9=0,78$ \\
\hline $\begin{array}{l}\text { Carbet } \\
\text { St-Pierre } \\
\text { Morne Vert- } \\
\text { Prêcheur }\end{array}$ & $9 / 12=0,75$ & $10 / 12=0,80$ & $8 / 12=0,75$ \\
\hline $\begin{array}{l}\text { Basse-Pointe- } \\
\text { Lorrain }\end{array}$ & $10 / 13=0,77$ & $10 / 13=0,77$ & $9 / 13=0,69$ \\
\hline Sainte-Marie & $5 / 21=0,24$ & $15 / 21=0,71$ & $7 / 21=0,33$ \\
\hline Trinité & $3 / 4=0,75$ & $1 / 4=0,25$ & $1 / 4=0,25$ \\
\hline Morne-Rouge & $8 / 18=0,44$ & $16 / 18=0,89$ & $13 / 18=0,72$ \\
\hline Gros-Morne & $4 / 7=0,57$ & $3 / 7=0,43$ & $3 / 7=0,43$ \\
\hline
\end{tabular}

quatre sérums ont été prélevés mais seulement 250 étaient accompagnés de fiches complètes.

Pour $B$. bovis et $B$. bigemina, les séroprévalences sont très corrélées entre elles et 83 p. 100 des bovins infectés par $B$. bigemina le sont aussi par $B$. bovis. Les bovins Brahman et croisés sont les plus infectés. Les communes du Lamentin, du François, Sainte-Anne, Morne Rouge, Saint-Denis, Saint-Joseph et Belle Fontaine ont les taux les plus élevés.

A. marginale : les races Charolais et Holstein sont les plus infectées. Les communes de Sainte-Luce, Ducos, Rivière-Salée et Diamant présentent les plus forts taux d'infection. L'infection est plus fréquente dans les troupeaux où n'existent pas d'Amblyomma.

Les résultats sérologiques par communes sont reportés sur les cartes 1, 2 et 3.

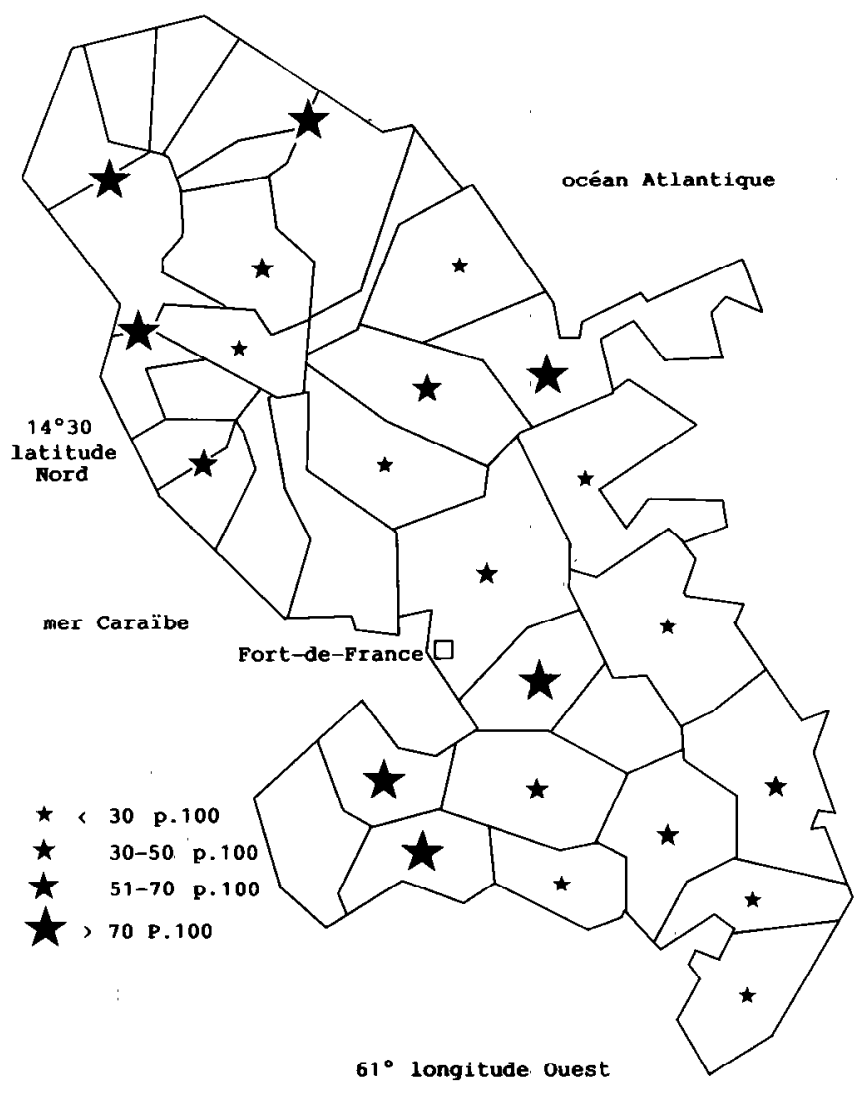

Carte I : Distribution par communes des bovins séropositifs vis-à-vis d'A. marginale.

\section{Comparaison des deux Card tests}

Sur 250 sérums testés, 108 sont agglutinés par l'antigène américain et 106 par l'antigène cubain.

Sur les 108 sérums positifs avec l'antigène américain, 105 sont positifs également avec l'antigène cubain (97 p. 100). Un seul sérum est positif avec l'antigène cubain et négatif avec l'antigène américain.

\section{Frottis}

Sur 80 frottis de sang et 13 frottis de cerveau prélevés dans 31 élevages, on a décelé : 3 cas de Babesia bovis (sang) ; 3 cas de B. bovis (cerveau) ; 4 cas d'Anaplasma marginale ; 1 cas de Theileria mutans.

Tous les frottis ont été réalisés dans le sud du département et uniquement sur des bovins importés (Holstein, Brahman, Charolais, Limousin).

Les six cas de babésiose ont été observés sur des bovins de race Holstein ; les quatre cas d'anaplasmose sur un taureau Limousin (mort), deux vaches laitières et une vache Brahman. Theileria mutans a été observé sur une vache Holstein. 


\section{Alonso E. Camus J. Rodriguez Diego L. Bertaudière J.C. Tatareau J.M. Liabeuf}

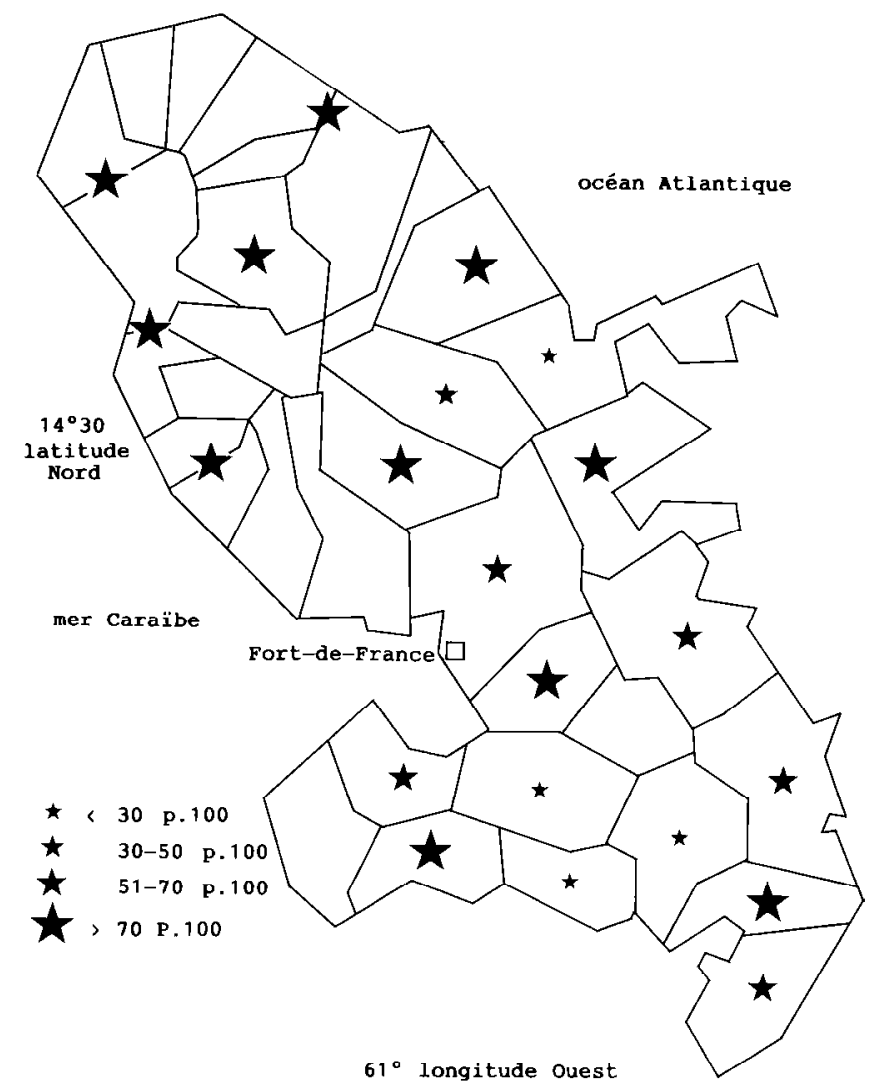

Carte 2 : Distribution par communes des bovins séropositifs vis-à-vis de Babesia bovis.

\section{DISCUSSION}

\section{Enquête sérologique}

La séroprévalence de Babesia bovis apparaît plus élevée dans le nord que dans le sud de l'île (72 p. 100 contre 55 p. 100). La différence va dans le même sens pour $B$. bigemina et $A$. marginale mais elle n'est pas significative.

La lutte contre la tique $A$. variegatum ne semble pas diminuer l'incidence des babésioses mais seulement celle de l'anaplasmose. Dans les trois communes du nord (SainteAnne, Marin, Vauclin) qui subissent un détiquage hebdomadaire depuis 10 ans, la séroprévalence des babésioses est de 61 p. 100 pour B. bovis et de 51 p. 100 pour $B$. bigemina contre 50 p. 100 et 46 p. 100 respectivement dans les autres communes du sud. Par contre, la séroprévalence de l'anaplasmose n'est que de 29 p. 100 dans les trois communes considérées, comparée à 49 p. 100 pour les autres communes du sud. Il est possible que l'usage intensif d'acaricide ait sélectionné des souches de Boophilus microplus résistantes mais ait diminué l'incidence de diptères piqueurs vecteurs de l'anaplasmose.

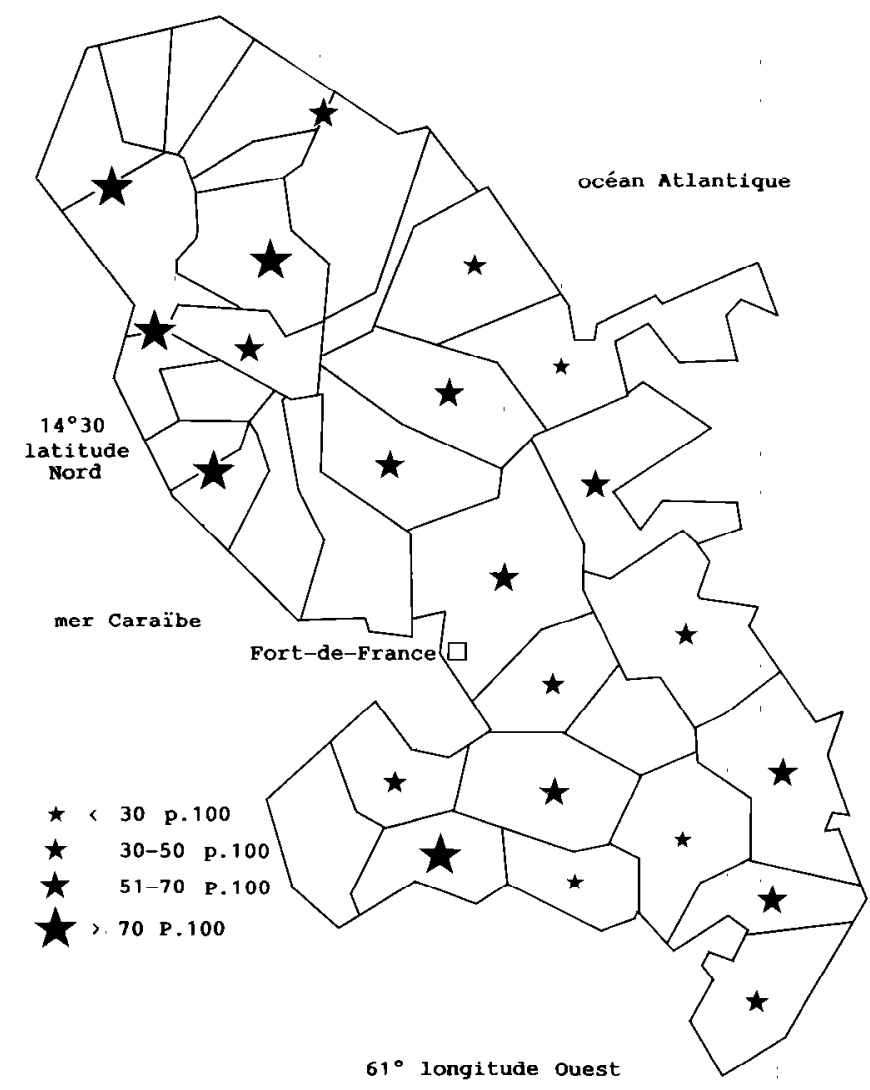

Carte 3 : Distribution par communes des bovins séropositifs vis-à-vis de Babesia bigemina.

Les bovins Créole sont apparemment beaucoup moins infectćs par les babćsioscs que les autres (DS 1 p. 100). II est possible aussi que les anticorps atteignent un taux moins élevé et persistent moins longtemps que chez les bovins moins rustiques. Le taux d'animaux sérologiquement positifs vis-à-vis des babésioses augmente avec l'âge jusqu'à 3-4 ans puis diminue.

L'absence de corrélation ( $r=0,24 \mathrm{NS}$ ), contrairement aux observations de HABICH et al. (9) et de DE RIOS et al. (6) en Argentine et TECLAW et al. (13) au Mexique, entre la séroprévalence de l'anaplasmose et de la babésiose à $B$. bovis pourrait indiquer que le vecteur de l'anaplasmose n'est sans doute pas uniquement Boophilus microplus mais également un (ou des) diptère(s) piqueur(s)' ; les Tabanides sont pratiquement absents du département, en revanche les stomoxes ou les Haematobia, voire les moustiques, pourraient jouer le rôle de vecteurs. Ce point devra être précisé par des enquêtes entomologiques suivies de recherches d'anaplasmes par sondes ADN ; cependant des résultats positifs ne prouveraient pas une transmission.

A partir des résultats sérologiques obtenus, peut-on estimer les caractéristiques de la situation épizootiologique pour l'anaplasmose et les babésioses en Martinique? 
Une extrapolation peut être tentée. La stabilité (ou l'instabilité) épizootiologique est déduite du pourcentage de bovins infectés entre l'âge de 6 et 12 mois (11) : avant 6 mois, les anticorps peuvent être d'origine colostrale, après 12 mois, les anticorps peuvent traduire, non pas une, mais plusieurs infections successives.

Si l'on suppose que le pourcentage de bovins séropositifs âgés de moins d'un an est représentatif de la population, malgré le faible nombre d'animaux de l'échantillon,

$I$ = pourcentage de bovins infectés

lo $=61$ p. 100 pour $B$. bovis ; $\mathrm{li}=48$ p. 100 pour $B$. bigemina ; la $=42$ p. 100 pour $A$. marginale

selon la formule de MAHONEY et ROSS (11).

$I=1-e^{-h t}$,

où h est le taux d'inoculation et t l'âge en jours des veaux.

Pour un âge moyen de neuf mois : ho $=0,0034 ; \mathrm{hi}=$ 0,0026 ; ha $=0,0019$.

La situation épizootiologique est instable lorsque $h<$ 0,005 et le risque est maximum, lorsque $h$ est compris entre 0,005 et 0,0005 . C'est apparemment le cas pour les trois parasitoses considérées, en Martinique.

Cette extrapolation devra être vérifiée en testant un plus grand nombre d'animaux dans la classe d'âge 6-12 mois, et en vérifiant, si cela est possible, que les cas cliniques sont nombreux.

L'absence d'anticorps contre $T$. vivax signe certainement la disparition de la trypanosomose, confirmée par l'absence de maladie clinique et l'absence de parasites sur les frottis de sang examinés. Le même phénomène a été observé en Guadeloupe où, après quelques années de présence (1926 à 1929) (7), T. vivax a disparu. La trypanosomose n'a sans doute pas trouvé des conditions favorables à son établissement dans ces îles : vecteur mécanique trop rare (taons, stomoxes), densité de bétail insuffisante...

\section{Comparaison des deux Card tests}

Élaborés avec des antigènes d'origine différente, les deux tests donnent des résultats tout à fait comparables. Le Card test reste un des deux tests de diagnostic officiel de l'anaplasmose aux États-Unis (avec fixation du complément) bien qu'il soit maintenant ancien (2). Dans une étude comparative, le card test a entraîné $2 \mathrm{p}$. 100 de réactions faussement positives et 16 p. 100 de réactions faussement négatives (8). Autrement dit le Card test sous-estime le pourcentage de bovins infectés. Dans l'étude, le pourcentage global réel serait d'environ 60 p. 100 et non 43 p. 100 d'infections.

Cependant, ce test facile et bon marché apparaît suffisant dans le cadre d'une enquête préliminaire.

\section{Frottis}

La plus grande sensibilité des races importées aux hémoparasitoses est, une fois de plus, confirmée.

La présence de Theileria mutans repose la question de son pouvoir pathogène : le parasite est-il pathogène ou n'est-il qu'un germe de sortie ? C'est la première observation de ce parasite en Martinique. II était déjà connu en Guadeloupe (14).

Comme en Guadeloupe et en Guyane, Babesia bovis apparaît sous forme clinique, contrairement à $B$. bigemina ( $E$. CAMUS, non publié) : quelle est l'incidence réelle de $B$. bigemina, aussi répandue que $B$. bovis?

Aucun des 13 frottis de cerveau ne présentait de colonies de Cowdria ruminantium. Cette observation renforce la présomption d'absence de cowdriose en Martinique; la présence d'environ 10 p. 100 d'animaux séropositifs (4) est sans doute à porter au compte de réactions croisées, puisqu'aucun cas clinique n'a jamais été rapporté et que l'inoculation de 500 tiques adultes Amblyomma variegatum, récoltées sur des bovins dans tout le sud du département et injectées à des chèvres sensibles, n'a pas entraîné l'apparition de cowdriose (CAMUS, non publié).

\section{CONCLUSION}

Babésioses et anaplasmoses bovines sont largement répandues en Martinique. Les séroprévalences élevées font supposer une situation épizootiologique instable pour ces trois hémoparasitoses mais une enquête complémentaire est nécessaire pour confirmer ce fait et en tirer des conclusions pratiques en matière de vaccination ou de traitement.

Le faible nombre de frottis observés ne permet que des conclusions limitées : l'anaplasmose et la babésiose à $B$. bovis existent cliniquement sans que leur incidence réelle puisse être appréciée. T. mutans existe en Martinique.

Les deux Card tests préparés avec des antigènes d'origine différente présentent des résultats pratiquement identiques pour détecter les anticorps anti-Anaplasma marginale. La trypanosomsose à $T$ : vivax a disparu de Martinique, cliniquement et sérologiquement.

\section{REMERCIEMENTS}

Nous remercions tous les vétérinaires techniciens de la Direction des Services vétérinaires et de l'Établissement départemental de l'Élevage qui ont participé à l'enquête : Dr FOUQUET, MM. MANIKON, LAGRANCOURT, BOUCAIN, MONLOUIS, CULORE, PIERRE-LOUIS, CHONVILLE, TRIOLET, BÉREAU, THIANT, NOTEUIL, SINSEAU, SELOI et AMORY. 


\section{Alonso E. Camus J. Rodriguez Diego L. Bertaudière J.C. Tatareau J.M. Liabeuf}

ALONSO (M.), CAMUS (E.), RODRIGUEZ DIEGO (J.), BERTAUDIERE (L.), TAT AREAU (J.C.), LIABEUF (J.M.). Present situation of bovine haemoparasitosis in Martinique (French West Indies). Revue Elev. Méd. vét. Pays trop., 1992, 45 (1) : 9-14

A serological survey using indirect immunofluorescence (IFAT) for bovine babesiosis (Babesia bovis and $B$. bigemina) and card test for anaplasmosis, indicates that these haemoparasites are widespread in Martinique. The high prevalences ( $62 \%$ for $B$. bovis, $52 \%$ for $B$. bigemina and $43 \%$ for Anaplasma marginale) lead to the hypothesis of an unstable epizootic situation for these three haemoparasitic diseases. However, the number of smears examined was too low to evaluate their clinical incidence. Both the American and Cuban card tests gave very similar results in the detection of antibodies to $\mathrm{A}$. marginale. Theileria mutans is described for the first time in Martinique. Trypanosomosis (Trypanosoma vivax) has disappeared from Martinique, on clinical and serological evidence. Key words : Babesia bovis - Babesia bigemina - Anaplasma marginale - Theileria mutans Trypanosoma vivax - Serology - Immunodiagnosis - Card test Martinique (French West Indies).
ALONSO (M.), CAMUS (E.), RODRIGUEZ DIEGO (J.), BERTAUDIERE (L.), TATAREAU (J.C.), LIABEUF (J.M.). Situación actual de los hemoparásitos bovinos en La Martinica (Antillas francesas). Revue Élev. Méd. vét. Pays trop., 1992, 45 (1) : 9-14

Una encuesta serológica llevada a cabo mediante inmunofluorescencia indirecta, para las babesiosis bovinas (Babesia bovis y $B$. bigemina) y por aglutinación, para la anaplasmosis bovina, indica que estos hemoparásitosis se encuentran ampliamente distribuídos en La Martinica. Las prevalencias séricas elevadas (62 p. 100 para $B$. bovis, 52 p. 100 para $B$. bigemina y 43 p. 100 para Anaplasma marginale) representan una situación epizootiológica inestable para estos tres hemoparásitos, pero el bajo número de frotis observados no permite una apreciación de la incidencia clínica. Los dos "card test" (americano y cubano) presentan resultados practicamente identicos en Ia detección de anticuerpos anti $A$. marginale. Theileria mutans se señala por primera vez en La Martinica. Con respecto a la tripanosomiasis (Trypanosoma vivax), se indica que esta ha desaparecido clínica y serológicamente de la isla. Palabras claves : Babesia bovis - Babesia bigemina - Anaplasma marginale - Theileria mutans - Trypanosoma vivax - Serología - Inmunodiagnóstico - Card test - (La) Martinica.

1. ALONSO (M.), BLANDINO (T.), LARRAMENDI (R.), MORALES (J.A.). Aplicacion de la aglutinacion en tarjeta en el diagnostico serologico de la anaplasmosis. XI Réunion ALPA. La Habana, Cuba, 1988.

2. AMERAULT (T.E.), OBY (T.O.). A rapid agglutination test for bovine anaplasmosis. J. Am. vet. Med. Assoc., 1968, 153 : 1828 1834.

3. BARRÉ (N.), MATHERON (G.), LEFEVRE (P.C.), LE GOFF (C.), ROGEZ (B.), ROGER (F.), MARTINEZ (D.), SHEIKBOUDOU (C.). La dermatophilose des bovins à Dermatophilus congolensis dans les Antilles françaises. I. Caractéristiques des lésions et de la réponse sérologique. Revue Élev. Méd. vét. Pays trop., 1988, 41 (2) : 129-138.

4. CAMUS (E.). Étude épidémiologique de la cowdriose à Cowdria ruminantium en Guadeloupe. Maisons-Alfort, IEMVT, 1989. 169 p. (Etudes et synthèses de l'IEMVT n ${ }^{\circ} 33$ )

5. CAROUGEAU (M.). Trypanosomiase bovine à la Martinique. Bull. Soc. Path. exot., 1929. P. $246-247$.

6. DE RIOS (L.G.), AGUIRRE (D.H.), GAIDO (A.B.). Infection naturelle par Anaplasma marginale chez les deux troupeaux de bovins avec différents niveaux d'infestation par la tique Boophilus microplus. Revue Élev. Méd. vét. Pays trop., 1990,43 (4) : $447-$ 452.

7. FABRE (H.J.A.). Note complémentaire sur la trypanosomomiase bovine à la Guadeloupe. Bull. Soc. Path. exot., 1929, 22 : 668669 .

8. GONZALEZ (G.E.), LONG (R.F.), TODOROVIC (R.A.). Comparison of the complement-fixation, indirect fluorescent antibody and card agglutination tests for the diagnosis of bovine anaplasmosis. Am. J. Vet. Res., 1978, 39 (9) : 1538-1541.

9. HABICH (G.E.), DE RIOS (L.G.), HADANI (A.), CONDRON (R.J.), DE HAAN (L.), BROADBENT (D.W.). Estudios sobre sanidad animal en el noreste argentino. VTII. Prevalencia de animales con anticuerpos sericos contra Babesia bovis y Anaplasma marginale en tambos de Catamara, Salta y Tucuman. Revue Méd. vét.(Bs. As.), 1982, $63: 316-329$.

10. LANCELOT (R.). La trypanosomose bovine à Trypanosoma vivax en Guyane française. Contribution à l'étude clinique et épidémiologique. Thèse doct. vét., Créteil, 1988. $116 \mathrm{p}$.

11. MAHONEY (D.F.), ROSS (D.R.). Epizootiological factors in the control of bovine babesiosis. Aust. Vet. J., 1972, 48 : 292-298

12. MOREL (P.C.). Étude sur les tiques du bétail en Guadcloupc et Martiniquc. II. Agents pathogènes transmis par les tiques. Revue Elev. Méd. vét. Pays trop., 1967, 20 (2) : 291-299.

13. TECLAW (R.F.), ROMO (S.), GARCIA (Z.), WAGNER (G.G.). A serological survey for anaplasmosis in cattle in the Mexican States of Nuevoleon, Tamaulipas and Coahuita using the card test. Prév. Vet. Med., 1985, 3: 417-425.

14. UILENBERG (G.), CAMUS (E.), BARRÉ (N.). Existence en Guadeloupe (French West Indies) de Theileria mutans et de Theileria velifera (Sporozoa, Theileriidae). Revue Élev. Méd. vét. Pays trop., 1983, 36 (3) : 261-264. 